Research Article

\title{
Permanence in Multispecies Nonautonomous Lotka-Volterra Competitive Systems with Delays and Impulses
}

\author{
Xiaomei Feng, ${ }^{1,2}$ Fengqin Zhang, ${ }^{2}$ Kai Wang, ${ }^{3}$ and Xiaoxia $\mathrm{Li}^{2}$ \\ ${ }^{1}$ College of Mathematics and System Sciences, Xinjiang University, Urumqi 830046, China \\ 2 Department of Mathematics, Yuncheng University, Yuncheng 044000, China \\ ${ }^{3}$ Department of Medical Engineering and Technology, Xinjiang Medical University, Urumqi 830011, China
}

Correspondence should be addressed to Kai Wang, wangkaimath@sina.com

Received 10 November 2011; Accepted 2 February 2012

Academic Editor: Zhen Jin

Copyright (c) 2012 Xiaomei Feng et al. This is an open access article distributed under the Creative Commons Attribution License, which permits unrestricted use, distribution, and reproduction in any medium, provided the original work is properly cited.

This paper studies multispecies nonautonomous Lotka-Volterra competitive systems with delays and fixed-time impulsive effects. The sufficient conditions of integrable form on the permanence of species are established.

\section{Introduction}

In this paper, we consider the nonautonomous $n$-species Lotka-Volterra type competitive systems with delays and impulses

$$
\begin{gathered}
x_{i}^{\prime}(t)=x_{i}(t)\left[a_{i}(t)-b_{i}(t) x_{i}(t)-\sum_{j=1}^{n} a_{i j}(t) x_{j}\left(t-\tau_{i j}(t)\right)\right], \quad t \neq t_{k} \\
x_{i}\left(t_{k}^{+}\right)=h_{i k} x_{i}\left(t_{k}\right), \quad i=1,2, \ldots, n, k=1,2, \ldots
\end{gathered}
$$

where $x_{i}(t)$ represents the population density of the $i$ th species at time $t$, the functions $a_{i}(t)$, $b_{i}(t), a_{i j}(t)$, and $\tau_{i j}(t)(i, j=1,2, \ldots, n)$ are bounded and continuous functions defined on $R_{+}=[0,+\infty), a_{i j}(t) \geq 0, b_{i}(t) \geq 0, \tau_{i j}(t) \geq 0$ for all $t \in R_{+}$, and impulsive coefficients $h_{i k}$ for any $i=1,2, \ldots, n$ and $k=1,2, \ldots$ are positive constants. 
In particular, when the delays $\tau_{i j}(t) \equiv 0$ for all $t \in R_{+}$and $i, j=1,2, \ldots, n$, then the system (1.1) degenerate into the following nondelayed non-autonomous $n$-species Lotkavolterra system

$$
\begin{gathered}
x_{i}^{\prime}(t)=x_{i}(t)\left[a_{i}(t)-\sum_{j=1}^{n} b_{i j}(t) x_{j}(t)\right], \quad t \neq t_{k}, \\
x_{i}\left(t_{k}^{+}\right)=h_{i k} x_{i}\left(t_{k}\right), \quad i=1,2, \ldots, n, k=1,2, \ldots,
\end{gathered}
$$

where $b_{i i}(t)=b_{i}(t)+a_{i i}(t)$ and $b_{i j}(t)=a_{i j}(t)$ for $i, j=1,2, \ldots, n$ and $i \neq j$. For system (1.2), the author establish some new sufficient condition on the permanence of species and global attractivity in [1].

As we well know, systems like (1.1) and (1.2) without impulses are very important in the models of multispecies populations dynamics. Many important results on the permanence, extinction, global asymptotical stability for the two species or multi-species nonautonomous Lotka-Volterra systems and their special cases of periodic and almost periodic systems can be found in [2-14] and the references therein.

However, owing to many natural and man-made factors (e.g., fire, flooding, crop-dusting, deforestation, hunting, harvesting, etc.), the intrinsic discipline of biological species or ecological environment usually undergoes some discrete changes of relatively short duration at some fixed times. Such sudden changes can often be characterized mathematically in the form of impulses. In the last decade, much work has been done on the ecosystem with impulsive(see [1, 15-21] and the reference therein). Specially, the following system is considered in [22]:

$$
\begin{gathered}
x_{i}^{\prime}(t)=x_{i}(t)\left[a_{i}(t)-b_{i i}(t) x_{i}(t)-\sum_{j=1, j \neq i}^{n} \int_{-\infty}^{0} k_{j}(s) x_{j}(t+s) \mathrm{d} s\right], \quad t \neq t_{k}, \\
x_{i}\left(t_{k}^{+}\right)=h_{i k} x_{i}\left(t_{k}\right), \quad i=1,2, \ldots, n, k=1,2, \ldots
\end{gathered}
$$

The author establish some new sufficient conditions on the permanence of species and global attractivity for system (1.3). However, the effect of discrete delays on the possibility of species survival has been an important subject in population biology. We find that infinite delays are considered in the system (1.3). In this paper, it is very meaningful that discrete delays are proposed in the impulsive system (1.1).

\section{Preliminaries}

Let $\tau=\sup \left\{\tau_{i j}(t), t \geq 0, i, j=1,2, \ldots, n\right\}$. We define $C^{n}[-\tau, 0]$ the Banach space of bounded continuous function $\phi:[-\tau, 0] \rightarrow R^{n}$ with the supremum norm defined by:

$$
\|\phi\|_{c}=\sup _{-\tau \leq s \leq 0}|\phi(s)|
$$

where $\phi=\left(\phi_{1}, \phi_{2}, \ldots, \phi_{n}\right)$, and $|\phi(s)|=\sum_{i=1}^{n}\left|\phi_{i}(s)\right|$. Define $C_{+}^{n}[-\tau, 0]=\left\{\phi=\left(\phi_{1}, \phi_{2}, \ldots, \phi_{n}\right) \in\right.$ $C^{n}[-\tau, 0]: \phi_{i}(s) \geq 0$, and $\phi_{i}(0) \geq 0$ for all $s \in[-\tau, 0]$ and $\left.i=1,2, \ldots, n\right\}$. 
Motivated by the biological background of system (1.1), we always assume that all solutions $\left(x_{1}(t), x_{2}(t), \ldots, x_{n}(t)\right)$ of system (1.1) satisfy the following initial condition:

$$
x_{i}(s)=\phi_{i}(s) \quad \forall s \in[-\tau, 0], i=1,2, \ldots, n,
$$

where $\phi=\left(\phi_{1}, \phi_{2}, \ldots, \phi_{n}\right) \in C_{+}^{n}[-\tau, 0]$.

It is obvious that the solution $\left(x_{1}(t), x_{2}(t), \ldots, x_{n}(t)\right)$ of system (1.1) with initial condition (2.2) is positive, that is, $x_{i}(t)>0(i=1,2, \ldots, n)$ on the interval of the existence and piecewise continuous with points of discontinuity of the first kind $t_{k}(k \in N)$ at which it is left continuous, that is, the following relations are satisfied:

$$
x_{i}\left(t_{k}^{-}\right)=x_{i}\left(t_{k}\right), \quad x_{i}\left(t_{k}^{+}\right)=h_{i k} x_{i}\left(t_{k}\right), \quad i=1,2, \ldots, n, k \in N .
$$

For system (1.1), we introduce the following assumptions:

$\left(\mathrm{H}_{1}\right)$ functions $a_{i}(t), b_{i}(t), a_{i j}(t)$ and $\tau_{i j}(t)$ are bounded continuous on $[0,+\infty]$, and $b_{i}(t)$, $a_{i j}(t)$ and $\tau_{i j}(t)(i, j=1,2, \ldots, n)$ are nonnegative for all $t \geq 0$.

$\left(\mathrm{H}_{2}\right)$ for each $1 \leq i \leq n$, there are positive constants $\omega_{i}>0$ such that

$$
\liminf _{t \rightarrow \infty}\left(\int_{t}^{t+\omega_{i}} b_{i}(s) \mathrm{d} s\right)>0
$$

and the functions

$$
h_{i}(t, \mu)=\sum_{t \leq t_{k}<t+\mu} \ln h_{i k}
$$

are bounded for all $t \in R_{+}$and $\mu \in\left[0, \omega_{i}\right]$.

First, we consider the following impulsive logistic system

$$
\begin{gathered}
x^{\prime}(t)=x(t)[\alpha(t)-\beta(t) x(t)], \quad t \neq t_{k}, \\
x\left(t_{k}^{+}\right)=h_{k} x\left(t_{k}\right), \quad k=1,2, \ldots,
\end{gathered}
$$

where $\alpha(t)$ and $\beta(t)$ are bounded and continuous functions defined on $R_{+}, \beta(t) \geq 0$ for all $t \in R_{+}$, and impulsive coefficients $h_{k}$ for any $k=1,2, \ldots$ are positive constants. We have the following results.

Lemma 2.1. Suppose that there is a positive constant $\omega$ such that

$$
\begin{gathered}
\liminf _{t \rightarrow \infty}\left(\int_{t}^{t+w} \beta(s) \mathrm{d} s\right)>0, \\
\liminf _{t \rightarrow \infty}\left(\int_{t}^{t+w} \alpha(s) \mathrm{d} s+\sum_{t \leq t_{k}<t+\omega} \ln h_{k}\right)>0,
\end{gathered}
$$


and function

$$
h(t, \mu)=\sum_{t \leq t_{k}<t+\omega} \ln h_{k}
$$

is bounded on $t \in R_{+}$and $\mu \in[0, \omega]$. Then we have

(a) there exist positive constants $m$ and $M$ such that

$$
m \leq \liminf _{t \rightarrow \infty} x(t) \leq \limsup _{t \rightarrow \infty} x(t) \leq M,
$$

for any positive solution $x(t)$ of system (2.6);

(b) $\lim _{t \rightarrow \infty}\left(x^{(1)}(t)-x^{(2)}(t)\right)=0$ for any two positive solutions $x^{(1)}(t)$ and $x^{(2)}(t)$ of system (2.6).

The proof of Lemma 2.1 can be found as Lemma 2.1 in [1] by Hou et al.

On the assumption $\left(\mathrm{H}_{2}\right)$, we firstly have the following result.

Lemma 2.2. If assumption $\left(\mathrm{H}_{2}\right)$ holds, then there exist constants $d>0$ and $D>0$ such that for any $t_{2} \geq t_{1} \geq 0$

$$
\left|\sum_{t_{1} \leq t_{k}<t_{2}} \ln h_{i k}\right| \leq d\left(t_{2}-t_{1}\right)+D, \quad i=1,2, \ldots, n
$$

The proof of Lemma 2.2 is simple, we hence omit it here.

\section{Main Results}

Let $x_{i 0}(t)$ be some fixed positive solution of the following impulsive logistic systems as the subsystems of system (1.1):

$$
\begin{gathered}
x_{i}^{\prime}(t)=x_{i}(t)\left[a_{i}(t)-b_{i}(t) x_{i}(t)\right], \quad t \neq t_{k}, \\
x_{i}\left(t_{k}^{+}\right)=h_{i k} x_{i}\left(t_{k}\right), \quad k=1,2, \ldots
\end{gathered}
$$

On the permanence of all species $x_{i}(i=1,2, \ldots, n)$ for system (1.1), we have the following result.

Theorem 3.1. Suppose that assumptions $\left(\mathrm{H}_{1}\right)-\left(\mathrm{H}_{2}\right)$ hold. If there exist positive constants $\omega_{i}$ such that for each $1 \leq i \leq n$ :

$$
\liminf _{t \rightarrow \infty}\left(\int_{t}^{t+\omega_{i}}\left(a_{i}(s)-\sum_{j \neq i}^{n} a_{i j}(s) x_{j 0}\left(s-\tau_{i j}(s)\right)\right) \mathrm{d} s+\sum_{t \leq t_{k}<t+\omega_{i}} \ln h_{i k}\right)>0,
$$


and the functions

$$
h_{i}(t, \mu)=\sum_{t \leq t_{k}<t+\mu} \ln h_{i k}
$$

are bounded for all $t \in R_{+}$and $\mu \in\left[0, \omega_{i}\right]$. Then the system (1.1) is permanent, that is, there are positive constants $\gamma>0$ and $M>0$ such that

$$
r \leq \liminf _{t \rightarrow \infty} x_{i}(t) \leq \limsup _{t \rightarrow \infty} x_{i}(t) \leq M, \quad i=1,2, \ldots, n,
$$

for any positive solution $x(t)=\left(x_{1}(t), x_{2}(t), \ldots, x_{n}(t)\right)$ of system $(1.1)$.

Proof. Let $x(t)=\left(x_{1}(t), x_{2}(t), \ldots, x_{n}(t)\right)$ be any positive solution of system (1.1). We first prove that the components $x_{i}(i=1,2, \ldots, n)$ of system (1.1) are bounded. From assumption $\left(\mathrm{H}_{1}\right)$ and the $i$ th equation of system (1.1), we have

$$
\begin{gathered}
x_{i}^{\prime}(t) \leq x_{i}(t)\left[a_{i}(t)-b_{i}(t) x_{i}(t)\right], \quad t \neq t_{k}, \\
x_{i}\left(t_{k}^{+}\right)=h_{i k} x_{i}\left(t_{k}\right), \quad k \in N .
\end{gathered}
$$

by the comparison theorem of impulsive differential equation, we have

$$
x_{i}(t) \leq y_{i}(t), \quad \forall t \geq 0,
$$

where $y_{i}(t)$ is the solution of (3.1) with initial value $y_{i}(0)=x_{i}(0)$. From the condition (3.2), we directly have

$$
\liminf _{t \rightarrow \infty}\left(\int_{t}^{t+\omega_{i}} a_{i}(s) \mathrm{d} s+\sum_{t \leq t_{k}<t+\omega_{i}} \ln h_{i k}\right)>0, \quad i=1,2, \ldots, n .
$$

Hence, from conclusion (a) of Lemma 2.1, we can obtain a constant $M_{i 1}>0$, and there is a $T_{i 1}>0$ such that $y_{i}(t)<M_{i 1}$ for all $t \geq T_{i 1}$. Let $M=\max _{1 \leq i \leq n}\left\{M_{i 1}\right\}$ and $T_{1}=\max _{1 \leq i \leq n}\left\{T_{i 1}\right\}$, we have

$$
x_{i}(t) \leq M, \quad \forall t \geq T_{1}, i=1,2, \ldots, n .
$$

Hence, we finally have

$$
\limsup _{t \rightarrow \infty} x(t) \leq M
$$

Next, we prove that there is a constant $\gamma>0$ such that

$$
\liminf _{t \rightarrow \infty} x(t) \geq \gamma, \quad i=1,2, \ldots, n
$$


For any $t_{1}$ and $t_{2}$ directly from system (1.1), we have

$$
x_{i}\left(t_{1}\right)=x_{i}\left(t_{2}\right) \exp \left(\int_{t_{2}}^{t_{1}}\left[a_{i}(t)-b_{i}(t) x_{i}(t)-\sum_{j=1}^{n} a_{i j}(t) x_{j}\left(t-\tau_{i j}(t)\right)\right] \mathrm{d} t+\sum_{t_{2} \leq t_{k} \leq t_{1}} \ln h_{i k}\right) .
$$

From condition (3.2), we can choose constants $0<\varepsilon<1$ small enough and $T_{2}>0$ large enough such that

$$
\int_{t}^{t+\omega_{i}}\left(a_{i}(s)-\left[b_{i}(s)+a_{i i}(s)\right] \varepsilon-\sum_{j \neq i}^{n} a_{i j}(s)\left[x_{j 0}\left(s-\tau_{i j}(s)\right)+\varepsilon\right]\right) \mathrm{d} s+\sum_{t \leq t_{k}<t+\omega_{i}} \ln h_{i k}>\varepsilon,
$$

for all $t \geq T_{2}$ and $i=1,2, \ldots, n$. Considering (3.5), by the comparison theorem of impulsive differential equation and the conclusion (b) of Lemma 2.1., we obtain for the above $\varepsilon \geq 0$ that there is a $T_{3}>T_{2}$ such that

$$
x_{i}(t) \leq x_{i 0}(t)+\varepsilon \quad \forall t \geq T_{3}, i=1,2, \ldots, n,
$$

where $x_{i 0}(t)$ is a globally uniformly attractive positive solution of system (3.1).

Claim 1. There is a constant $\eta>0$ such that $\lim \sup _{t \rightarrow \infty} x_{i}(t)>\eta(i=1,2, \ldots, n)$ for any positive solution $x(t)=\left(x_{1}(t), x_{2}(t), \ldots, x_{n}(t)\right)$ of system (1.1). In fact, if Claim 1 is not true, then there is an integer $k \in\{1,2, \ldots, n\}$ and a positive solution $x(t)=\left(x_{1}(t), x_{2}(t), \ldots, x_{n}(t)\right)$ of system (1.1) such that

$$
\limsup _{t \rightarrow \infty} x_{k}(t)<\varepsilon
$$

Hence, there is a constant $T_{4}>T_{3}$ such that

$$
x_{k}(t)<\varepsilon \quad \forall t \geq T_{4} .
$$

On the other hand, by (3.13) there is a $T_{5} \geq T_{4}$ such that

$$
x_{i}(t) \leq x_{i 0}(t)+\varepsilon \quad \forall t \geq T_{5},
$$


where $i=1,2, \ldots, n$ and $i \neq k$. By (3.11) and (3.16), we obtain

$$
\begin{aligned}
& x_{k}(t)=x_{k}\left(T_{5}+\tau\right) \exp \left(\int_{T_{5}+\tau}^{t}\left[a_{k}(s)-b_{k}(s) x_{k}(t)-\sum_{j=1}^{n} a_{k j}(s) x_{j}\left(s-\tau_{i j}(s)\right)\right] \mathrm{d} s\right. \\
&+\left.\sum_{T_{5}+\tau \leq t_{k} \leq t} \ln h_{k k}\right) \\
& \geq x_{k}\left(T_{5}+\tau\right) \exp \left(\int_{T_{5}+\tau}^{t}\left[a_{k}(s)-\left(b_{k}(s)+a_{k k}(s)\right) \varepsilon-\sum_{j=1, j \neq k}^{n} a_{i j}(s)\left(x_{j 0}\left(s-\tau_{i j}(s)\right)+\varepsilon\right)\right] \mathrm{d} s\right. \\
&\left.+\sum_{T_{5}+\tau \leq t_{k} \leq t} \ln h_{k k}\right),
\end{aligned}
$$

for all $t \geq T_{5}+\tau$. Thus, from (3.12) we finally obtain $\lim _{t \rightarrow \infty} x_{k}(t)=\infty$, which lead to a contradiction.

Claim 2. There is a constant $\gamma>0$ such that $\lim _{\inf _{t \rightarrow \infty}} x_{i}(t)>\gamma(i=1,2, \ldots, n)$ for any positive solution of system (1.1).

If Claim 2 is not true, then there is an integer $k \in\{1,2, \ldots, n\}$ and a sequence of initial function $\left\{\phi_{m}\right\} \subset C_{+}[-\tau, 0]$ such that

$$
\liminf _{t \rightarrow \infty} x_{k}\left(t, \phi_{m}\right)<\frac{\eta}{m^{2}} \quad \forall m=1,2, \ldots
$$

where constant $\eta$ is given in Claim 1 . By Claim 1 , for every $m$ there are two time sequences $s_{q}^{(m)}$ and $t_{q}^{(m)}$, satisfying:

$$
0<s_{1}^{(m)}<t_{1}^{(m)}<s_{2}^{(m)}<t_{2}^{(m)}<\cdots<s_{q}^{(m)}<t_{q}^{(m)}<\cdots, \quad \lim _{q \rightarrow \infty} s_{q}^{(m)}=\infty
$$

such that

$$
\begin{gathered}
x_{k}\left(s_{q}^{(m)}, \phi_{m}\right) \geq \frac{\eta}{m}, \quad x_{k}\left(t_{q}^{(m)}, \phi_{m}\right) \leq \frac{\eta}{m^{2}}, \\
\frac{\eta}{m^{2}} \leq x_{k}\left(t, \phi_{m}\right) \leq \frac{\eta}{m} \quad \forall t \in\left(s_{q}^{(m)}, t_{q}^{(m)}\right) .
\end{gathered}
$$


From the above proof, there is a constant $T^{(m)} \geq T_{2}$ such that $x_{i}\left(t, \phi_{m}\right)<M(i=1,2, \ldots, n)$ for all $t \geq T^{(m)}$. Further, there is an integer $K_{1}^{(m)}>0$ such that $s_{q}^{(m)}>T^{(m)}$ for all $q>K_{1}^{(m)}$. From (3.11) and lemma 2.2., we can obtain

$$
\begin{aligned}
x_{k}\left(t_{q}^{(m)}, \phi_{m}\right) & \geq x_{k}\left(s_{q}^{(m)}, \phi_{m}\right) \exp \left(\int_{s_{q}^{(m)}}^{t_{q}^{(m)}}\left[a_{k}(s)-b_{k}(s) M-\sum_{j=1}^{n} a_{k j}(s) M\right] \mathrm{d} s+\sum_{s_{q}^{(m)} \leq t_{k} \leq t_{q}^{(m)}} \ln h_{k k}\right) \\
& \geq x_{k}\left(s_{q}^{(m)}, \phi_{m}\right) \exp \left(-\left(r_{1}+d\right)\left(t_{q}^{(m)}-s_{q}^{(m)}\right)-D\right),
\end{aligned}
$$

where $r_{1}=\sup _{t \geq 0}\left\{\left|a_{i}(t)\right|+b_{i}(t) M+\sum_{j=1}^{n} a_{i j}(t) M\right\}$. Consequently, from (3.20) we have

$$
t_{q}^{(m)}-s_{q}^{(m)} \geq \frac{\ln m-D}{r_{1}+d} \quad \forall q>K_{1}^{(m)}
$$

By (3.12), there is a large enough $P>0$ such that for all $t \geq T_{2}, a \geq P$ and $a \in\left[l w_{i},(l+1) w_{i}\right)$ and $i=1,2, \ldots, n$, then, we obtain

$$
\begin{aligned}
& \int_{t}^{t+a}\left(a_{i}(s)-\left[b_{i}(s)+a_{i i}(s)\right] \varepsilon-\sum_{j \neq i}^{n} a_{i j}(s)\left[x_{j 0}\left(s-\tau_{i j}(s)\right)+\varepsilon\right]\right) \mathrm{d} s+\sum_{t \leq t_{k}<t+a} \ln h_{i k} \\
& =\int_{t}^{t+l w_{i}}\left(a_{i}(s)-\left[b_{i}(s)+a_{i i}(s)\right] \varepsilon-\sum_{j \neq i}^{n} a_{i j}(s)\left[x_{j 0}\left(s-\tau_{i j}(s)\right)+\varepsilon\right]\right) \mathrm{d} s+\sum_{t \leq t_{k}<t+l w_{i}} \ln h_{i k} \\
& \quad+\int_{t+l w_{i}}^{t+a}\left(a_{i}(s)-\left[b_{i}(s)+a_{i i}(s)\right] \varepsilon-\sum_{j \neq i}^{n} a_{i j}(s)\left[x_{j 0}\left(s-\tau_{i j}(s)\right)+\varepsilon\right]\right) \mathrm{d} s+\sum_{t+l w_{i} \leq t_{k}<t+a} \ln h_{i k} \\
& >l \varepsilon-r_{2} w_{i},
\end{aligned}
$$

where $r_{2}=\sup _{t \geq 0}\left\{\left|a_{i}(t)\right|+\left[b_{i}(t)+a_{i i}(t)\right] \varepsilon+\sum_{j \neq i}^{n} a_{i j}(s)\left[x_{j 0}\left(s-\tau_{i j}(s)\right)+\varepsilon\right]\right\}$. So, we choose $L=2+\left(r_{2} w_{i} / \varepsilon\right)$ such that for all $l>L$, we have

$$
\int_{t}^{t+a}\left(a_{i}(s)-\left[b_{i}(s)+a_{i i}(s)\right] \varepsilon-\sum_{j \neq i}^{n} a_{i j}(s)\left[x_{j 0}\left(s-\tau_{i j}(s)\right)+\varepsilon\right]\right) \mathrm{d} s+\sum_{t \leq t_{k}<t+a} \ln h_{i k}>\varepsilon .
$$

From (3.23), there is an integer $N_{0}$ such that for any $m>N_{0}$ and $q>K_{1}^{(m)}$, we have

$$
\frac{\eta}{m}<\varepsilon, \quad t_{q}^{(m)}-s_{q}^{(m)}>2 Q,
$$

where constant $Q>P+\tau$. 
So, when $m>N_{0}$ and $q>K_{1}^{(m)}$, for any $t \in\left[s_{q}^{(m)}+Q+\tau, t_{q}^{(m)}\right]$, from (3.11), (3.21), (3.25), and (3.26) we can obtain

$$
\begin{aligned}
x_{k}\left(t_{q}^{(m)}, \phi_{m}\right)= & x_{k}\left(s_{q}^{(m)}+Q+\tau, \phi_{m}\right) \\
& \times \exp \left(\int_{s_{q}^{(m)}+Q+\tau}^{t_{q}^{(m)}}\left[a_{k}(t)-b_{k}(t) x_{k}\left(t, \phi_{m}\right)-\sum_{j=1}^{n} a_{k j}(t) x_{j 0}\left(t-\tau_{k j}(t)\right), \phi_{m}\right] \mathrm{d} t\right. \\
& \left.+\sum_{s_{q}^{(m)}+Q+\tau \leq t_{k} \leq t_{q}^{(m)}} \ln h_{k k}\right)
\end{aligned}
$$

Consequently, from (3.20) and (3.25) it follows

$$
\begin{aligned}
& \frac{\eta}{m^{2}} \geq \frac{\eta}{m^{2}} \\
& \times \exp \left(\int_{s_{q}^{(m)}+Q+\tau}^{t_{q}^{(m)}}\left[a_{k}(t)-\left(b_{k}(t)+a_{k k}(t)\right) \varepsilon-\sum_{j=1, j \neq k}^{n} a_{k j}(t) x_{j 0}\left(t-\tau_{k j}(t)\right)+\varepsilon\right] \mathrm{d} t\right. \\
& \left.+\sum_{s_{q}^{(m)}+Q+\tau \leq t_{k} \leq t_{q}^{(m)}} \ln h_{k k}\right) \\
& >\frac{\eta}{m^{2}} \text {. }
\end{aligned}
$$

This leads to a contradiction. Therefore, Claim 2 is true. This completes the proof.

When system (1.1) degenerates into the periodic case, then we can assume that there is a constant $\omega>0$ and an integer $q>0$ such that $a_{i}(t+\omega)=a_{i}(t), b_{i}(t+\omega)=b_{i}(t), a_{i j}(t+$ $\omega)=a_{i j}(t), t_{k+q}=t_{k}+\omega$ and $h_{i k+q}=h_{i k}$ for all $t \in R_{+}, k=1,2, \ldots$ and $i, j=1,2, \ldots, n$. From Remarks 2.3 and 2.4 in [1], we can see the fixed positive solution $x_{j 0}$ of system (3.1) can be chosen to be the $\omega$-periodic solution of system (3.1). Therefore, as a consequence of Theorem 3.1. we have the following result.

Corollary 3.2. Suppose that system (1.1) is w-periodic and for each $i=1,2, \ldots, n$,

$$
\begin{gathered}
\int_{0}^{\omega} b_{i}(s) \mathrm{d} s>0, \\
\int_{0}^{\omega}\left(a_{i}(s)-\sum_{j \neq i}^{n} a_{i j}(s) x_{j 0}\left(s-\tau_{i j}(s)\right)\right) \mathrm{d} s+\sum_{k=1}^{q} \ln h_{i k}>0 .
\end{gathered}
$$

Then, system (1.1) is permanent. 


\section{Numerical Example}

In this section, we will give an example to demonstrate the effectiveness of our main results. We consider the following two species competitive system with delays and impulses:

$$
\begin{aligned}
x_{1}^{\prime}(t)=x_{1}(t)\left[a_{1}(t)-b_{1}(t) x_{1}(t)\right. & \left.-a_{11}(t) x_{1}\left(t-\tau_{11}(t)\right)-a_{12}(t) x_{2}\left(t-\tau_{12}(t)\right)\right], \quad t \neq t_{k} \\
x_{2}^{\prime}(t)=x_{2}(t)\left[a_{2}(t)-b_{2}(t) x_{2}(t)\right. & \left.-a_{21}(t) x_{1}\left(t-\tau_{21}(t)\right)-a_{22}(t) x_{2}\left(t-\tau_{22}(t)\right)\right], \\
x_{1}\left(t_{k}^{+}\right) & =h_{1 k} x_{1}\left(t_{k}\right), \quad k=1,2, \ldots \\
x_{2}\left(t_{k}^{+}\right) & =h_{2 k} x_{2}\left(t_{k}\right), \quad k
\end{aligned}
$$

We take $a_{1}(t)=2, a_{2}(t)=b_{1}(t)=b_{2}(t)=a_{11}(t)=a_{12}(t)=a_{22}(t)=1, a_{21}=1-|\sin (\pi / 2) t|$, $\tau_{i j}(t)=2, h_{1 k}=e^{-1}, h_{2 k}=e, t_{k}=k$. Obviously, system (4.1) is periodic with period $\omega=2$. For $q=2$, we have $t_{k+q}=t_{k}+\omega, h_{1 k+q}=h_{1 k}$ and $h_{2 k+q}=h_{2 k}$ for all $k=1,2, \ldots$. Consider the following impulsive logistic systems as the subsystems of system (4.1):

$$
\begin{aligned}
& x_{1}^{\prime}(t)=x_{1}(t)\left(2-x_{1}(t)\right), \quad t \neq k \\
& x_{2}^{\prime}(t)=x_{2}(t)\left(1-x_{2}(t)\right), \quad \\
& x_{1}\left(t^{+}\right)=e^{-1} x_{1}\left(t_{k}\right), \quad t=k .
\end{aligned}
$$

According to the formula in [1], we can obtain that subsystem (4.2) has a unique globally asymptotically stable positive 2-periodic solution $\left(x_{10}(t), x_{20}(t)\right)$, which can be expressed in following form:

$$
\begin{aligned}
& x_{10}(t)=\frac{2 x_{10}}{x_{10}+\left(2-x_{10}\right) e^{-2(t-k)}}, \quad t \in[k, k+1), k=0,1,2, \ldots, \\
& x_{20}(t)=\frac{x_{20}}{x_{20}+\left(1-x_{20}\right) e^{-(t-k)}}, \quad t \in[k, k+1), k=0,1,2, \ldots,
\end{aligned}
$$

where $x_{10}=\left(2\left(e^{-0.2}-e^{-2}\right) /\left(1-e^{-2}\right)\right)$ and $x_{20}=\left(e-e^{-1}\right) /\left(1-e^{-1}\right)$. Since

$$
\begin{aligned}
& \int_{0}^{\omega}\left(a_{1}(t)-a_{12}(t) x_{20}\left(t-\tau_{12}(t)\right)\right) \mathrm{d} t+\sum_{k=1}^{q} \ln h_{1 k} \\
& \quad=2 \int_{0}^{1}\left(2-\frac{x_{20}}{x_{20}+\left(1-x_{20}\right) e^{-(t-2)}}\right) \mathrm{d} t+\sum_{k=1}^{2} \ln h_{1 k} \\
& \quad \approx 1.5244 \\
& \int_{0}^{\omega}\left(a_{2}(t)-a_{21}(t) x_{10}\left(t-\tau_{21}(t)\right)\right) \mathrm{d} t+\sum_{k=1}^{q} \ln h_{2 k} \\
& \quad=2 \int_{0}^{1}\left(1-\left(1-\sin \frac{\pi}{2} t\right) \frac{2 x_{10}}{x_{10}+\left(2-x_{10}\right) e^{-2(t-2)}}\right) \mathrm{d} t+\sum_{k=1}^{2} \ln h_{2 k} \\
& \quad \approx 3.8398
\end{aligned}
$$




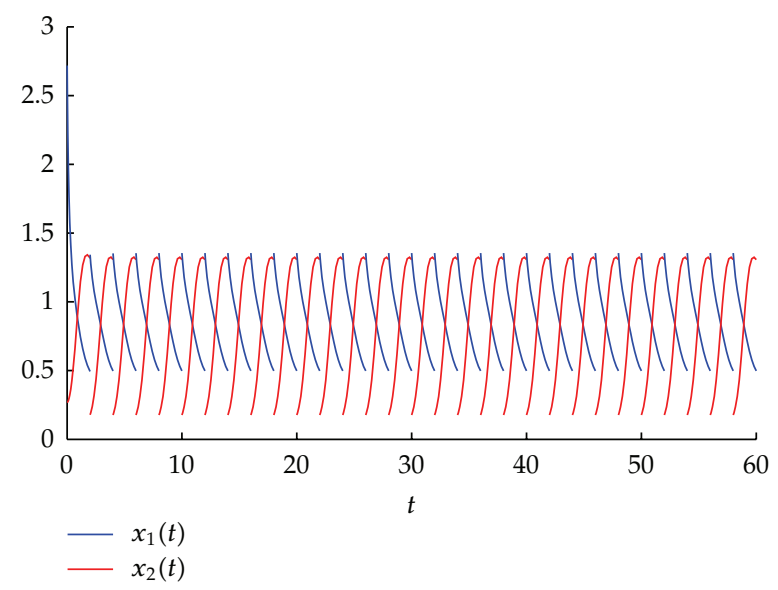

Figure 1: Time series of $x_{1}(t)$ and $x_{2}(t)$.

we obtain that all conditions in Corollary 3.2 for system (1.1) holds. Therefore, from Theorem 3.1. we see that system (1.1) is permanent (see Figure 1).

\section{Acknowledgments}

This paper was supported by the National Sciences Foundation of China (11071283), the Sciences Foundation of Shanxi (2009011005-3), the Young foundation of Shanxi province (no. 2011021001-1), research project supported by Shanxi Scholarship Council of China (2011093), the Major Subject Foundation of Shanxi, and Doctoral Scientific Research fund of Xinjiang Medical University.

\section{References}

[1] J. Hou, Z. Teng, and S. Gao, "Permanence and global stability for nonautonomous N-species LotkaValterra competitive system with impulses," Nonlinear Analysis, vol. 11, no. 3, pp. 1882-1896, 2010.

[2] S. Ahmad and M. Rama Mohana Rao, "Asymptotically periodic solutions of $n$-competing species problem with time delays," Journal of Mathematical Analysis and Applications, vol. 186, no. 2, pp. 559$571,1994$.

[3] H. I. Freedman and S. G. Ruan, “Uniform persistence in functional-differential equations," Journal of Differential Equations, vol. 115, no. 1, pp. 173-192, 1995.

[4] H. I. Freedman and J. H. Wu, "Periodic solutions of single-species models with periodic delay," SIAM Journal on Mathematical Analysis, vol. 23, no. 3, pp. 689-701, 1992.

[5] G. Seifert, "On a delay-differential equation for single specie population variations," Nonlinear Analysis, vol. 11, no. 9, pp. 1051-1059, 1987.

[6] X.-Z. He and K. Gopalsamy, "Persistence, attractivity, and delay in facultative mutualism," Journal of Mathematical Analysis and Applications, vol. 215, no. 1, pp. 154-173, 1997.

[7] K. Gopalsamy, Stability and Oscillations in Delay Differential Equations of Population Dynamics, vol. 74, Kluwer Academic, Dordrecht, The Netherlands, 1992.

[8] Y. Kuang, Delay Differential Equations, with Applications in Population Dynamics, Academin Press, New York, NY, USA, 1965.

[9] B. Lisena, "Global attractivity in nonautonomous logistic equations with delay," Nonlinear Analysis, vol. 9, no. 1, pp. 53-63, 2008. 
[10] Z. Teng and Z. Li, "Permanence and asymptotic behavior of the $N$-species nonautonomous LotkaVolterra competitive systems," Computers \& Mathematics with Applications, vol. 39, no. 7-8, pp. 107-116, 2000.

[11] S. Ahmad and A. C. Lazer, "On a property of nonautonomous Lotka-Volterra competition model," Nonlinear Analysis, vol. 37, no. 5, pp. 603-611, 1999.

[12] Y. Muroya, "Permanence of nonautonomous Lotka-Volterra delay differential systems," Applied Mathematics Letters, vol. 19, no. 5, pp. 445-450, 2006.

[13] Z. Teng, "Persistence and stability in general nonautonomous single-species Kolmogorov systems with delays," Nonlinear Analysis, vol. 8, no. 1, pp. 230-248, 2007.

[14] J. Wu and X.-Q. Zhao, "Permanence and convergence in multi-species competition systems with delay," Proceedings of the American Mathematical Society, vol. 126, no. 6, pp. 1709-1714, 1998.

[15] B. Liu, Z. Teng, and W. Liu, "Dynamic behaviors of the periodic Lotka-Volterra competing system with impulsive perturbations," Chaos, Solitons and Fractals, vol. 31, no. 2, pp. 356-370, 2007.

[16] L. Nie, Z. Teng, L. Hu, and J. Peng, "The dynamics of a Lotka-Volterra predator-prey model with state dependent impulsive harvest for predator," Biosystems, vol. 98, pp. 67-72, 2009.

[17] Z. Jin, H. Maoan, and L. Guihua, "The persistence in a Lotka-Volterra competition systems with impulsive," Chaos, Solitons and Fractals, vol. 24, no. 4, pp. 1105-1117, 2005.

[18] Z. Jin, M. Zhien, and H. Maoan, "The existence of periodic solutions of the $n$-species Lotka-Volterra competition systems with impulsive," Chaos, Solitons and Fractals, vol. 22, no. 1, pp. 181-188, 2004.

[19] Y. Xia, "Global analysis of an impulsive delayed Lotka-Volterra competition system," Communications in Nonlinear Science and Numerical Simulation, vol. 16, no. 3, pp. 1597-1616, 2011.

[20] W. Wang, J. Shen, and Z. Luo, "Partial survival and extinction in two competing species with impulses," Nonlinear Analysis, vol. 10, no. 3, pp. 1243-1254, 2009.

[21] L. Nie, J. Peng, Z. Teng, and L. Hu, "Existence and stability of periodic solution of a Lotka-Volterra predator-prey model with state dependent impulsive effects," Journal of Computational and Applied Mathematics, vol. 224, no. 2, pp. 544-555, 2009.

[22] H. Hu, K. Wang, and D. Wu, "Permanence and global stability for nonautonomous N-species LotkaVolterra competitive system with impulses and infinite delays," Journal of Mathematical Analysis and Applications, vol. 377, no. 1, pp. 145-160, 2011. 


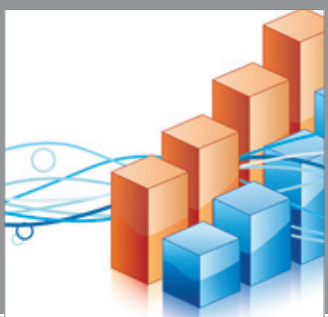

Advances in

Operations Research

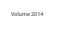

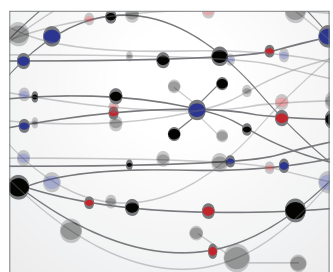

\section{The Scientific} World Journal
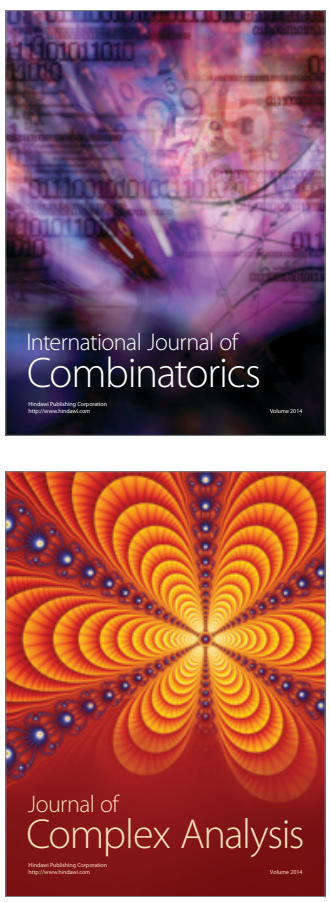

International Journal of

Mathematics and

Mathematical

Sciences
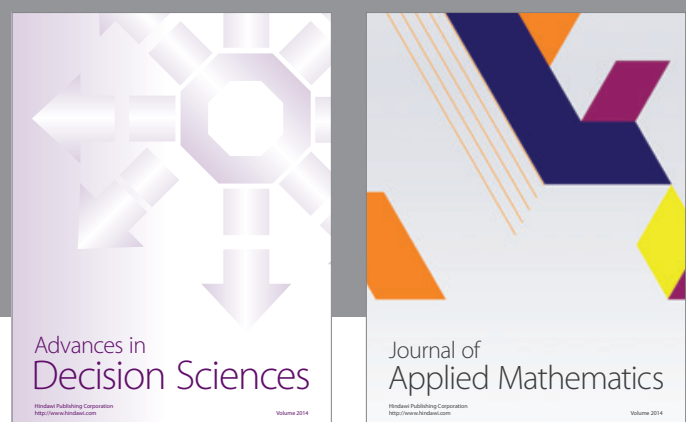

Journal of

Applied Mathematics
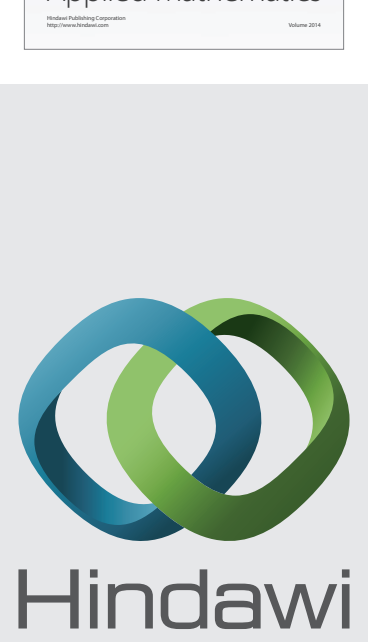

Submit your manuscripts at http://www.hindawi.com
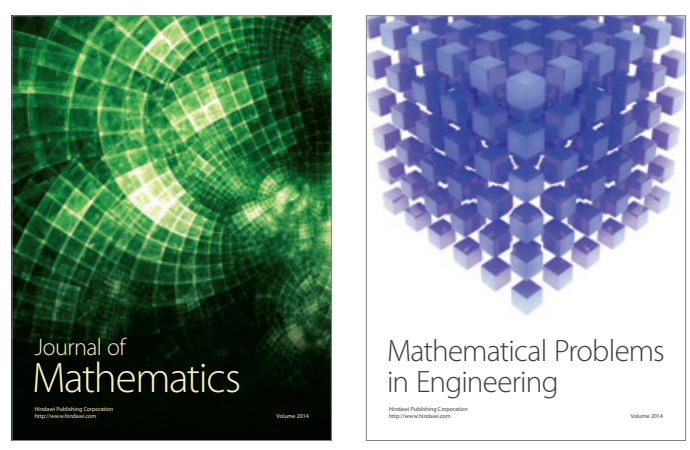

Mathematical Problems in Engineering
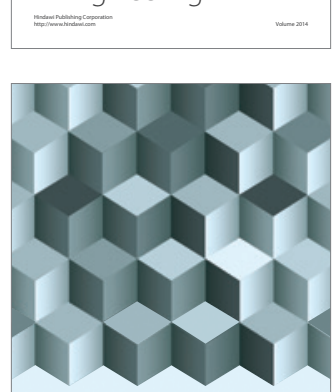

Journal of

Function Spaces
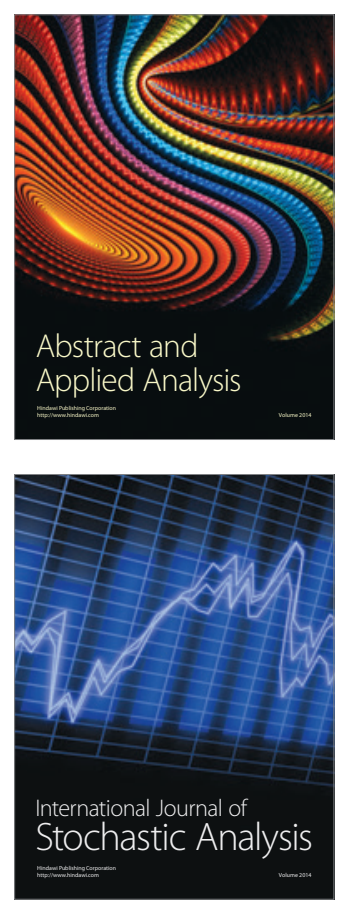

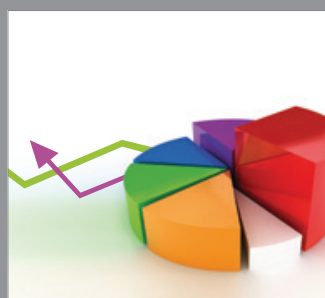

ournal of

Probability and Statistics

Promensencen
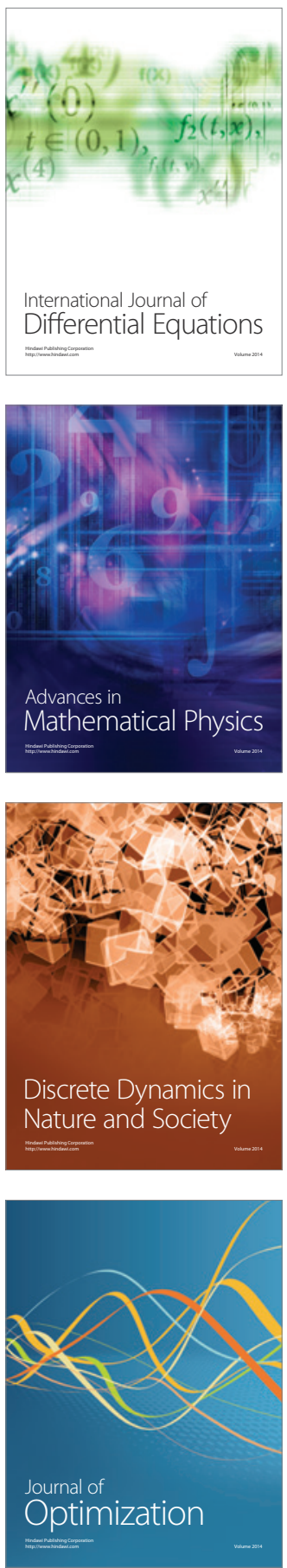